\title{
2
}

\section{VBR Video in ATM without Frame-Buffering: Influence of a Periodic Correlation Function on QOS Parameters*}

\author{
Christoph Herrmann \\ Communication Networks, Aachen University of Technology \\ Kopernikusstr.16, D-52056 Aachen, Germany, E-Mail: chh@dfv.rwth-aachen.de
}

Analytical models for video sources have in common that the resulting correlation function has an exponential decay. This property is realistic in case of a frame-buffered video source. Without frame buffering the correlation function was shown by measurements to have periodically occuring peaks (called "recorrelations") that decay exponentially. In order to characterize a video source with recorrelations, in [15], [7] a (modified) ARMAmodel was proposed, which allows to adjust the correlation function of the process to that of the measured video source. However, the ARMA model can only be used in computer simulations, since analytical solutions of queues with ARMA input are not known to date.

This paper investigates the DMAP ${ }^{[X]}$ (Discrete Markovian Arrival process with an independent random variable $\mathrm{X}$ modulating the batch size) for characterization of a video source with recorrelations. Choosing the matrices appropriately it is possible to gain recorrelations in the correlation function (of the number of arrivals in a test-interval or of the interarrival times). By means of the $\mathrm{DMAP}_{1}^{[X]}+\mathrm{DMAP}_{2} / \mathrm{D} / 1 / \mathrm{s}$ queue, models with periodic correlation function and those for the frame-buffered case are compared, and it turns out that negative values in the correlation function for small lags reduce loss probability and waiting time.

Keyword Codes: I.6.4, G.2.m, G.3

Keywords: ATM, Discrete-time Models for VBR Video, Periodic Correlation Function

\section{Introduction}

ATM is expected to allow transmission of video signals with more or less constant quality for the duration of that video connection, since statistical multiplexing permits variable bitrate. Constant bitrate codecs show the disadvantage that it is necessary to use a rougher quantizer as soon as the scene changes become too big [9], p.834, which can degrade the picture quality. Most investigations into the statistical nature of VBR video sources make the assumption that a full video frame is prebuffered and then transmitted in cells, so that the cell rate is constant for the duration of a frame [1] [16] [14] [12]. With this assumption, the (auto)correlation function of the bitrate, resp. of the number of cells generated in a sufficiently small test-interval resembles the negative exponential function, since periodic variations due to similarities between neighbouring lines (spatial correlation) or similarities in corresponding areas on consecutive video frames (temporal correlation) are absorbed. If there is no prebuffering, measurements [15] [7] have shown the

*This work was supported by the Deutsche Forschungsgemeinschaft within "Graduiertenkolleg Informatik \& Technik". 
periodic nature of the correlation function of the count of cells in a test-interval resulting from similarities in consecutive video frames. A (modified) ARMA model (AutoRegressive Moving Average) was used there in order to fit the mean, variance and the measured correlation function of the count of cells generated by the codec within the test-interval of 64 ATM-cells. For three different scenes generated by a Conditional Replenishment video codec, the correlation function was reconstructed.

So far there is no exact method for analyzing the behaviour of a queueing system fed by an ARMA-input and so the queueing performance of video can only be evaluated by computer simulations with all the big problems of the extremely rare events of loss for video (standardized tolerable loss probability: $10^{-9}$ ). For details about the ARMA-model see e.g. $[2,5]$

A characterization by means of the GMDP (Geometrically Modulated Deterministic Process) model in [4], which is analytically tractable, failed as stated by the authors, since (besides other reasons) the correlation function of the GMDP does not involve the periodic behaviour, which results if there is no prebuffering.

This paper investigates the DMAP ${ }^{[X]}$ source model (DMAP: Discrete Markovian Arrival Process) for rebuilding periodic correlation functions of the count of cells in a test-interval, as they were measured in [15] [7]. The DMAP ${ }^{[X]}$ (DMAP: Discrete Markovian Arrival process) is a special case of a DBMAP (Discrete Batch Markovian Arrival process), where the DMAP produces the arrival instants and the independent random variable $X$ determines the count of cells produced at that time instant. The batch random variable is used in order to model the count of cells generated in the test-interval as it was measured in [15] [7]. The periodicity of the correlation function can be captured by the model, if the Markov Chain of the phase transitions of the DMAP has periodic states. An analytically tractable representation of the video scenes, which involves at the same time a test-interval of 64 ATM cells, the measured batch size p.f. ${ }^{2}$, the same recorrelation peaks and a period of 325 test-intervals, could not be found. However, for periods shorter than 325 , it is possible to investigate the influence of the periodic correlation function in comparison with source models for frame-buffered video.

By means of the DBMAP/D/1/s finite buffer queue, models for the frame-buffered case [1] are compared with the case of no prebuffering. The properties of the DBMAP (the superposition $\mathrm{DMAP}_{1}^{[X]}+\mathrm{DMAP}_{2}$ is known to be a DBMAP) furthermore allow the superposition of different streams and the computation of per-stream quality of service (QOS) parameters. This is explained in section 4. The DBMAP/D/1/s finite buffer queue can be solved exactly for a deterministic service time equal to the time unit for the "Departure First" rule (The departing cell leaves before the arriving one enters) according to [1], and for the "Arrival First" rule (The arriving cell still sees the departing one) according to [17]. We used the "Departure First" rule.

Numerical results show that especially negative values of the correlation function within the first period reduce the QOS parameters in comparison with the frame-buffered case. This indicates that periodic variations in the correlation function are worth exploiting in statistical multiplexing, besides the disadvantage of additional delay for prebuffering, since cells are not generated before the whole frame has been stored. (This additional

${ }^{2}$ p.f. $=$ probability function 
delay would be $40 \mathrm{~ms}$ for the codec used in $[15,7]$, which corresponds, with a 48 bytes information field per cell, to about 1365 cells.) One reason for the relatively small cells in ATM was the reduction of packetizing delay and so the more natural approach with respect to the idea of ATM is sending video cells without prebuffering. In short: One should encode video in such a way that negative values of the correlation function occur for small lags.

\section{The Discrete Batch Markovian Arrival Process (DBMAP)}

We recall the definition of the DBMAP (Discrete time analogue of the Batch Markovian Arrival process due to Lucantoni [11]) given in [1]. Consider an $m$-state Markov chain (MC). A transition from state $S(t)=i$ to state $S(t+1)=j, t=0,1, \ldots$, happens according to the transition probabilities $\left[\mathbf{D}_{w}\right]_{i j}\left(\mathbf{D}_{w}\right.$ : transition matrix, $[\cdot]_{i j}$ : entry in the $i$-th row and $j$-th column) $w=0,1,2, \ldots, b_{\text {max }}$, and a transition is interpreted as an arrival of a batch of size $w$, if $w>0$ and there is no arrival if $w=0$. Thus the model generates interarrival times with batch arrivals possible, and successive interarrival times are not independent. In order to distinguish from other state random variables, the $m$ states of the DBMAP are usually referred to as phases. The MC has different types of transitions between two phases $i$ and $j$, thus extending the conventional definition of a MC. $\widetilde{\mathbf{D}}:=\sum_{w=0}^{\infty} \mathbf{D}_{w}$ is a stochastic matrix and represents the (conventional) MC which only considers the transitions between two phases no matter what type of transition. Let $\underline{\pi}$ denote the stationary phase probability vector $\underline{\pi} \widetilde{\mathbf{D}}=\underline{\pi}$. The arrival rate is given by $\underline{\pi} \sum_{v=1}^{b_{\max }} v \mathbf{D}_{v} \underline{e}, \underline{e}=(1, \ldots, 1)^{T}$.

In the special case of the DMAP (with only single arrivals, $b_{\max }=1$ ) it is the custom to write: $\mathbf{C}:=\mathbf{D}_{0}$, and $\mathbf{D}:=\mathbf{D}_{1}$.

In the following we focus on the special case of the $\mathrm{DMAP}^{[X]}$, where the independent process $X$ of i.i.d. random variables $X_{n}$ determines the size of a batch at the $n$-th arrival instant (generated by the DMAP) according to the probability function $x(v)=P\left\{X_{n}=v\right\}$. It is completely described by: $\mathbf{D}_{0}=\mathbf{C}$ and $\mathbf{D}_{w}=x(w) \cdot \mathbf{D}, \quad 1 \leq w \leq b_{\max }[1]$.

Furthermore the DMAP can be understood as a Semi-Markov process with the SemiMarkov kernel $\mathbf{q}(k):=\mathbf{C}^{k-1} \mathbf{D}$ and the p.f. of the interarrival times is given by $\underline{P} \mathbf{q}(k) \underline{e}$, with $\underline{P}=\frac{1}{\underline{\pi} \mathbf{D} \underline{\underline{e}}} \underline{\underline{D}} \mathbf{D}$ denoting the stationary solution of the MC $(\mathbf{I}-\mathbf{C})^{-1} \mathbf{D}$ embedded at arrival instants [8], [3].

\subsection{Counting process of the DBMAP}

According to [1] the counting process of a DBMAP is a discrete-time two-dimensional MC $\left\{(N(0 ; t], S(t)), t \in \mathbb{N}_{0}\right\}$, where $N(0 ; t]$ denotes the count of arrivals in the interval $(0, t], S(t) \in\{1,2, \ldots, m\}$ the phase of the process.

A transition from state $(v, i)$ to $(w, j)$ corresponds to an arrival of size $w-v$ and a change of the phase of the input process from $i$ to $j$. The corresponding state transition probability is

$P\{N(0 ; t+1]=w, S(t+1)=j \mid N(0 ; t]=v, S(t)=i\}=\left\{\begin{array}{ll}{\left[\mathbf{D}_{w-v}\right]_{i j}} & \text { for } w \geq v \\ 0 & \text { else }\end{array}\right.$.

We define: $P\{N(0 ; 0]=v, S(0)=i\}=\delta_{v, 0} \cdot P\{S(0)=i\}, \delta_{v, 0}$ : Kronecker symbol. Due to the Markov property it follows: 


$$
\begin{aligned}
& P\left\{N\left(0 ; t+t_{0}\right]=w, S\left(t+t_{0}\right)=j \mid N(0 ; t]=v, S(t)=i\right\}= \\
& =P\left\{N\left(0 ; t_{0}\right]=w-v, S\left(t_{0}\right)=j \mid N(0 ; 0]=0, S(0)=i\right\}
\end{aligned}
$$

and the probability of $w-v \geq 0$ arrivals within an interval of length $t_{0}$ only depends on $t_{0}$ and the phases at the beginning and at the end of this interval. In the following we use the abbreviation ( I: $m \times m$-matrix of unity ):

$$
\left[\mathbf{P}\left(w, t_{0}\right)\right]_{i j}:=P\left\{N\left(0 ; t_{0}\right]=w, S\left(t_{0}\right)=j \mid N(0 ; 0]=0, \quad S(0)=i\right\}, \quad \mathbf{P}(w, 0)=\delta_{0, w} \cdot \mathbf{I} .
$$

With the aid of the Markov property, the following recursion can be shown:

$\mathbf{P}(w, t+1)= \begin{cases}\mathbf{P}(0, t) \cdot \mathbf{D}_{0}=\mathbf{D}_{0}^{t+1} & \text { for } w=0 \\ \sum_{v=0}^{w} \mathbf{P}(v, t) \mathbf{D}_{w-v} & \text { for } w \geq 1\end{cases}$

By complete induction, the $z$-transform is found with the help of (2):

$$
\sum_{w=0}^{\infty} \mathbf{P}(w, t) z^{w}=\left(\sum_{w=0}^{b_{\max }} \mathbf{D}_{w} z^{w}\right)^{t} \quad t \geq 1
$$

For the special case of the $\operatorname{DMAP}^{[X]}$ it is:

$$
\sum_{w=0}^{\infty} \mathbf{P}(w, t) z^{w}=\left(\mathbf{C}+\sum_{w=1}^{b_{\max }} \mathbf{D}_{w} z^{w}\right)^{t}=(\mathbf{C}+\widehat{X}(z) \mathbf{D})^{t},
$$

where $\widehat{X}(z)=\sum_{v=1}^{\infty} x(v) z^{v}$ is the generating function of the batch size. With the identity

$$
\frac{d}{d z}(\mathbf{C}+\widehat{X}(z) \mathbf{D})^{n}=\sum_{i=1}^{n}(\mathbf{C}+\widehat{X}(z) \mathbf{D})^{i-1} \mathbf{D}(\mathbf{C}+\widehat{X}(z) \mathbf{D})^{n-i} \frac{d}{d z} \widehat{X}(z)
$$

the $k$-th moment $\sum_{v=0}^{\infty} v^{k} \mathbf{P}(v, t)$ can be computed by applying the limit theorem for $z \rightarrow 1$. The first two moments are $\left(\bar{x}^{(1)}:=\sum_{v=1}^{\infty} v x(v), \quad \bar{x}^{(2)}:=\sum_{v=1}^{\infty} v^{2} x(v)\right)$ :

$$
\begin{aligned}
& \sum_{v=1}^{\infty} v \mathbf{P}(v, t)=\bar{x}^{(1)} \cdot \sum_{i=1}^{t}(\mathbf{C}+\mathbf{D})^{i-1} \mathbf{D}(\mathbf{C}+\mathbf{D})^{t-i} \\
& \sum_{v=1}^{\infty} v^{2} \mathbf{P}(v, t)-\sum_{v=1}^{\infty} v \mathbf{P}(v, t)=\lim _{z \rightarrow 1} \frac{d^{2}}{d z^{2}} \sum_{v=0}^{\infty} \mathbf{P}(v, t) z^{v}= \\
& =\bar{x}^{(1)} \cdot \sum_{i=2}^{t}\left[\sum_{v=1}^{\infty} v \mathbf{P}(v, i-1)\right] \mathbf{D}(\mathbf{C}+\mathbf{D})^{t-i}+\bar{x}^{(1)} \cdot \sum_{i=1}^{t-1}(\mathbf{C}+\mathbf{D})^{i-1} \mathbf{D}\left[\sum_{v=1}^{\infty} v \mathbf{P}(v, t-i)\right]+ \\
& \quad+\left(\bar{x}^{(2)}-\bar{x}^{(1)}\right) \cdot \sum_{i=1}^{t}(\mathbf{C}+\mathbf{D})^{i-1} \mathbf{D}(\mathbf{C}+\mathbf{D})^{t-i}
\end{aligned}
$$




\subsection{Coefficient of correlation of the count of arrivals for the $\operatorname{DMAP}^{[X]}$}

In order to rebuild the correlation functions presented in $[15,7]$ by means of a $\operatorname{DMAP}^{[X]}$, the coefficient of correlation of ${ }^{\tau} \mathcal{N}_{t}:=N(t ; t+\tau]$ is now computed. ${ }^{\tau} \mathcal{N}_{t}$ represents the count of cells generated in the interval $(t ; t+\tau]$, and we need the coefficient of correlation of the count of arrivals in $(t ; t+\tau]$ and in the time-shifted interval $(t+h \tau ; t+(h+1) \tau]$ :

$$
\begin{aligned}
& \operatorname{Corr}(h)=\frac{\mathrm{E}\left[{ }^{\tau} \mathcal{N}_{t} \cdot{ }^{\tau} \mathcal{N}_{t+h \tau}\right]-\mathrm{E}^{2}\left[{ }^{\tau} \mathcal{N}_{t}\right]}{\mathrm{E}\left[{ }^{\tau} \mathcal{N}_{t}^{2}\right]-\mathrm{E}^{2}\left[\mathcal{N}_{t}\right]} \\
& \mathrm{E}\left[\mathcal{N}_{t} \cdot{ }^{\tau} \mathcal{N}_{t+h \tau}\right]=\underline{\pi}\left[\sum_{v=1}^{\infty} v \mathbf{P}(v, \tau)\right](\mathbf{C}+\mathbf{D})^{(h-1) \tau}\left[\sum_{w=1}^{\infty} w \mathbf{P}(w, \tau)\right] \underline{e}= \\
& =\left[\bar{x}^{(1)}\right]^{2} \underline{\pi}\left[\mathbf{D} \sum_{i=1}^{\tau}(\mathbf{C}+\mathbf{D})^{\tau-i}\right](\mathbf{C}+\mathbf{D})^{(h-1) \tau}\left[\sum_{i=1}^{\tau}(\mathbf{C}+\mathbf{D})^{i-1} \mathbf{D}\right] \underline{e} \\
& \mathrm{E}\left[{ }^{\tau} \mathcal{N}_{t}^{2}\right]=2\left[\bar{x}^{(1)}\right]^{2} \underline{\pi} \mathbf{D} \sum_{i=0}^{\tau-2} \sum_{\xi=0}^{i}(\mathbf{C}+\mathbf{D})^{\xi} \mathbf{D} \underline{e}+\bar{x}^{(2)} \tau \underline{\pi} \mathbf{D} \underline{e} \\
& \mathrm{E}\left[\mathcal{N}_{t}\right]=\bar{x}^{(1)} \tau \underline{\pi} \mathbf{D} \underline{e}
\end{aligned}
$$

Using the identities $\underline{\pi}(\mathbf{C}+\mathbf{D})=\underline{\pi}$ and $(\mathbf{C}+\mathbf{D}) \underline{e}=\underline{e}$ it is easily shown that (7) is invariant to an exchange of $\mathbf{C}$ and $\mathbf{D}$ so that there always exist two DMAPs with the same correlation function, but different arrival rates $\underline{\pi} \mathbf{D} \underline{e}$ and $1-\underline{\pi} \mathbf{D} \underline{e}$ (unless $\underline{\pi} \mathrm{D} \underline{e}=0.5$ ).

\section{Choosing $\mathrm{C}$ and $\mathrm{D}$ for a periodic correlation function}

For $\tau=1$ equation (8) reduces to $E\left[\mathcal{N}_{t} \cdot{ }^{1} \mathcal{N}_{t+h}\right]=\left[\bar{x}^{(1)}\right]^{2} \underline{\pi} \mathbf{D}(\mathbf{C}+\mathbf{D})^{h-1} \mathbf{D} \underline{e}$. Periodicity in Corr results, if the MC of the phase transition matrix $\mathbf{C}+\mathbf{D}$ contains periodic states (for MCs with periodic states see e.g. [3]), and the simplest choice (producing the biggest period with a minimum count of states) is the matrix for cyclic shifting

$$
\mathbf{C}+\mathbf{D}=\mathbf{M}_{c y c l}=\left(\begin{array}{ccccc}
0 & 1 & 0 & \cdots & 0 \\
& & \ddots & & \\
& & & \ddots & \\
& & & & 1 \\
1 & 0 & \cdots & & 0
\end{array}\right) \text {, }
$$

and this choice induces $\mathbf{D}=\operatorname{Diag}\left(d_{1}, d_{2}, \ldots, d_{m}\right) \cdot \mathbf{M}_{\text {cycl }}$, with $0 \leq d_{i} \leq 1,1 \leq i \leq m$. In the following the vector $\underline{d}:=\left(d_{1}, d_{2}, \ldots, d_{m}\right)$ is used to simplify notation. The stationary state probability $\underline{\pi}$ results in $\underline{\pi}=\left(\frac{1}{m}, \frac{1}{m}, \ldots, \frac{1}{m}\right)$. Finally it is:

$\mathrm{E}\left[{ }^{1} \mathcal{N}_{t} \cdot{ }^{1} \mathcal{N}_{t+h}\right]=\frac{\left[\bar{x}^{(1)}\right]^{2}}{m} \underline{d} \cdot\left[\mathbf{M}_{c y c l}\right]^{h} \cdot \underline{d}^{T}$

and

$$
\operatorname{Corr}(h)=\frac{m \underline{d} \cdot \mathbf{M}_{c y c l}^{h} \cdot \underline{d}^{T}-(\underline{d} \cdot \underline{e})^{2}}{\frac{\bar{x}^{(2)}}{\left[\bar{x}^{(1)}\right]^{2}} m \underline{d} \cdot \underline{e}-(\underline{d} \cdot \underline{e})^{2}}
$$


In order to get amplitudes which fall with higher values of the lag, it is possible to replace e.g. the first row in $\mathbf{M}_{c y c l e}$ by $(0, a, b, 0, \ldots, 0), a+b=1$. For $a=0.99$ the peak values fall with the factor of about 0.999, as it was measured in [15]. Then, of course, computation of Corr $(h)$ needs the general expression (7).

It was found very difficult to obtain a matrix $\mathbf{D}=\operatorname{Diag}\left(d_{1}, \ldots, d_{m}\right) \cdot \mathbf{M}_{\text {cycle }}$ in case $\tau>1$ so that the correlation function looked similar to the curves in Fig. 1 to 4 for $\tau=1$. For $\tau>1$ there are additional oscillations within one period, obviously due to the fact, that $\mathbf{M}_{\text {cycle }}^{\tau}$ performs a cyclic shift of $\tau$. The only case where exactly the same curves could be found was that of

$$
\mathrm{C}^{\prime}:=\left(\begin{array}{lllll}
\mathbf{0} & \mathrm{I} & \mathbf{0} & \cdots & 0 \\
\mathbf{0} & \mathbf{0} & \mathrm{I} & & \mathbf{0} \\
\vdots & & & \ddots & \vdots \\
\mathbf{0} & & & & \mathrm{I} \\
\mathrm{C} & 0 & 0 & \cdots & 0
\end{array}\right) \quad \mathrm{D}^{\prime}:=\left(\begin{array}{llll}
0 & 0 & \cdots & 0 \\
& & & \\
\vdots & & & \vdots \\
0 & 0 & & 0 \\
\mathrm{D} & 0 & \cdots & 0
\end{array}\right)
$$

with $\tau$ main rows and main columns. For this choice the first summand in (9) is equal to zero and (8) becomes equal to $\left[\bar{x}^{(1)}\right]^{2} \tau \underline{\pi} \mathbf{D M}_{\text {cycle }}^{h-1} \mathbf{D} \underline{e}$ with $\underline{\pi}=\left(\frac{1}{m \tau}, \ldots, \frac{1}{m \tau}\right)$, so that the same expression for Corr $(h)$ results. The possible interarrival times are integer multiples of $\tau$.

Exchanging C' and D' yields a DMAP with all integers possible as interarrival times and, of course, a higher arrival rate.

It should be noted that the amplitudes of Corr $(h)$ in (11) are often decreased considerably if $b_{\text {max }}>1$, since $\bar{x}^{(2)} \geq\left[\bar{x}^{(1)}\right]^{2}$.

\subsection{Correlation functions similar to those measured for real video scenes in the literature}

In $[15,7]$ the test-interval was chosen to be 64 ATM-cells long, and due to the minimum interarrival distance between cells of 7.63 cells, the maximum count of cells of that source within the above test-interval is about $b_{\max }=8$ [7], p. 286. Using the above mentioned matrices C' and 'D' with $\tau=64$ and $b_{\max }=8$ (one could even use the p.f. of the count of cells arriving in the test-interval as given in [15]) it would be possible to model the scenes rather closely by means of a DMAP ${ }^{[X]}$. However, since the period in those scenes is 325 (measurements in $[15,7]$ were finished before the standardization, therefore the authors used a cell length of $32+4$ bytes) it would be necessary to have $325 \cdot 64=20800$ states for one scene, and this is of course much too much if a queue were fed by such a process in order to analyse the behaviour of an ATM multiplexer (computations for the results given here used less than 400 phases for a superposition). Using the standardized cell length of $48+5$ bytes will not reduce the count of states considerably. A test-interval of 64 is necessary, if the multiplexer performance is to be analyzed by means of a discrete-time queue, which does not allow deterministic service times smaller than the time unit.

As to the magnitude of the peaks of the correlation function, it is interesting to mention that those measured for a test-interval of only 16 ATM cells were much lower than those for the 64 ATM cell test-interval [15], but additional oscillations occurred.

Nevertheless a comparison between conventional models for video sources with a correlation function of the bitrate which is negative exponential due to frame-buffering and 
Table 1 The values chosen for $d_{i}$, $1 \leq i \leq m$ for the three scenes with periodic correlation function (period: $m=20$, arrival rate: $0.345)$.

\begin{tabular}{|c||c|c|c|c|c|c|c|c|c|c|}
\hline & $d_{1}$ & $d_{2}$ & $d_{3}$ & $d_{4}$ & $d_{5}$ & $d_{6}$ & $d_{7}$ & $d_{8}$ & $d_{9}$ & $d_{10}$ \\
\hline \hline Scene1 & 0.2 & 0.15 & 0.1 & 0.05 & 0.5 & 0.1 & 0.3 & 0.05 & 0.05 & 0.05 \\
\hline Scene2 & 0.2 & 0.18 & 0.16 & 0.14 & 0.11 & 0.05 & 0.01 & 0.01 & 0.01 & 0.01 \\
\hline Scene3 & 0.6 & 0.4 & 0.4 & 0.3 & 0.3 & 0.2 & 0.15 & 0.15 & 0.15 & 0.1 \\
\hline \hline & $d_{11}$ & $d_{12}$ & $d_{13}$ & $d_{14}$ & $d_{15}$ & $d_{16}$ & $d_{17}$ & $d_{18}$ & $d_{19}$ & $d_{20}$ \\
\hline \hline Scene1 & 0.7 & 0.8 & 0.7 & 0.15 & 0.2 & 0.3 & 0.3 & 0.4 & 0.9 & 0.9 \\
\hline Scene2 & 0.01 & 0.01 & 0.4 & 0.4 & 0.7 & 0.9 & 0.9 & 0.9 & 0.9 & 0.9 \\
\hline Scene3 & 0.1 & 0.1 & 0.15 & 0.15 & 0.15 & 0.4 & 0.6 & 0.8 & 0.8 & 0.9 \\
\hline
\end{tabular}

stochastic processes with periodic correlation function is possible. Table 1 shows the values for $\underline{d}$ in order to get periodic correlation functions for $\tau=1$ and $b_{\max }=1$ as plotted (together with the correlation functions of superpositions) in Fig. 1 to 4 . Scene 1 resembles the head and shoulders scene and Scene 2 the low-flying aircraft scene in $[15,7]$, compare Fig. 1 and 2 with Fig. 11 in Appendix B. The parameters for the frame-buffered model are listed at the beginning of section 4.3 .

\subsection{Correlation function of a superposition}

Since the DBMAP is closed under superposition, the correlation function of two (or more) superimposed streams with some periodicity can be computed. Using the generating function the following expressions are obtained for the superposition of $\operatorname{DMAP}_{1}^{\left[X_{1}\right]}$ and $\operatorname{DMAP}_{2}^{\left[X_{2}\right]}$ given by $\left(\mathbf{C}^{(1)}, \mathbf{D}^{(1)}, x_{1}(v)\right)$ and $\left(\mathbf{C}^{(2)}, \mathbf{D}^{(2)}, x_{2}(v)\right)$, respectively $(\tau=1)$.

Note that $\mathrm{DMAP}_{1}^{\left[X_{1}\right]}+\mathrm{DMAP}_{2}^{\left[X_{2}\right]}$ is a DBMAP with $\mathbf{D}_{0}=\mathbf{C}^{(1)} \otimes \mathbf{C}^{(2)}$ and $\mathbf{D}_{v}=x_{1}(v)\left[\mathbf{D}^{(1)} \otimes\right.$ $\left.\mathbf{C}^{(2)}\right]+\sum_{\ell=1}^{v-1} x_{1}(\ell) x_{2}(v-\ell)\left[\mathbf{D}^{(1)} \otimes \mathbf{D}^{(2)}\right]+x_{2}(v)\left[\mathbf{C}^{(1)} \otimes \mathbf{D}^{(2)}\right](\otimes:$ Kronecker-product $)$.

$$
\begin{aligned}
& \sum_{v=1}^{\infty} v \mathbf{P}(v, 1)=\left(\mathbf{C}^{(1)}+\mathbf{D}^{(1)}\right) \otimes \mathbf{D}^{(2)} \bar{x}_{2}^{(1)}+\bar{x}_{1}^{(1)} \mathbf{D}^{(1)} \otimes\left(\mathbf{C}^{(2)}+\mathbf{D}^{(2)}\right) \\
& \mathrm{E}\left[\mathcal{N}_{t}{ }^{1} \mathcal{N}_{t+h}\right]=\underline{\pi} \sum_{v=1}^{\infty} v \mathbf{P}(v, 1) \cdot\left[\left(\mathbf{C}^{(1)}+\mathbf{D}^{(1)}\right) \otimes\left(\mathbf{C}^{(2)}+\mathbf{D}^{(2)}\right)\right]^{h-1} \cdot \sum_{v=1}^{\infty} v \mathbf{P}(v, 1) \underline{e}
\end{aligned}
$$

$$
\mathrm{E}\left[{ }^{1} \mathcal{N}_{t}^{2}\right]=\underline{\pi}\left[\left(\mathbf{C}^{(1)} \otimes \mathbf{D}^{(2)}\right) \bar{x}_{2}^{(2)}+\left(\mathbf{D}^{(1)} \otimes \mathbf{C}^{(2)}\right) \bar{x}_{1}^{(2)}+\right.
$$$$
\left.+\left(\mathbf{D}^{(1)} \otimes \mathbf{D}^{(2)}\right)\left(\bar{x}_{2}^{(2)}+2 \bar{x}_{1}^{(1)} \bar{x}_{2}^{(1)}+\bar{x}_{1}^{(2)}\right)\right] \underline{e}
$$

$\mathrm{E}\left[{ }^{1} \mathcal{N}_{t}\right]=\underline{\pi}\left(\left[\left(\mathbf{C}^{(1)}+\mathbf{D}^{(1)}\right) \otimes \mathbf{D}^{(2)}\right] \bar{x}_{2}^{(1)}+\left[\mathbf{D}^{(1)} \otimes\left(\mathbf{C}^{(2)}+\mathbf{D}^{(2)}\right)\right] \bar{x}_{1}^{(1)}\right) \underline{e}$

Fig. 1 to 4 also show superpositions of different scenes. It can be seen that the peak values in the superposition lie between the peak values of the superimposed scenes. The lag $h$ with the first negative value of the correlation function grows in the superposition.

\subsection{Correlation function of the interarrival times}

Measurements mostly take into account the count of cells generated in a test-interval or the bitrate. An interesting question is how the correlation function of the interarrival 


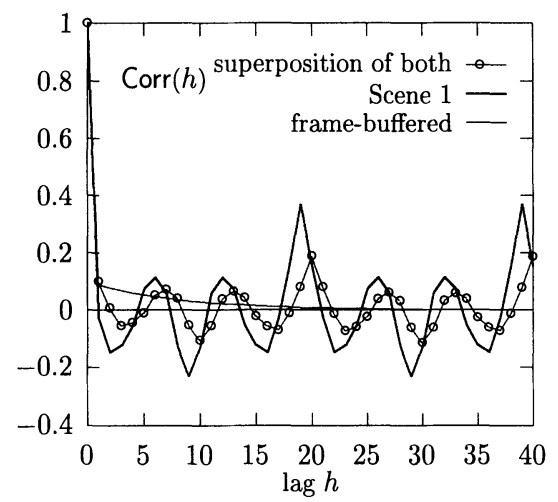

Fig. 1 Correlation functions: Scene 1, frame-buffered model and the superposition of both.

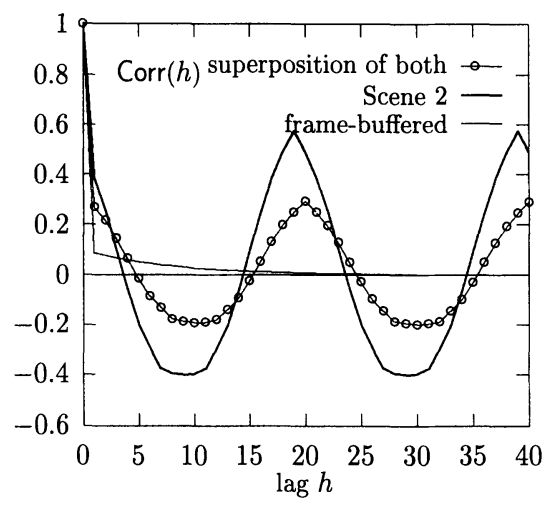

Fig. 2 Correlation functions: Scene 2, frame-buffered model and the superposition of both.

times looks like. In case of the DMAP this function can also be computed, as follows: Let $A_{n}$ denote the time between the $n$-th and the $(n+1)$-th arrival instant generated by a $\mathrm{DMAP}$ (or $\mathrm{DMAP}^{[X]}$ ). With $P\left\{A_{n}=t, A_{n+h}=t^{\prime}\right\}=\underline{P} \mathbf{q}(t)\left[(\mathbf{I}-\mathbf{C})^{-1} \mathbf{D}\right]^{h-1} \mathbf{q}\left(t^{\prime}\right) \underline{e}$ (see e.g. [8]) it is:

$$
\begin{aligned}
& \mathrm{E}\left[A_{n} \cdot A_{n+h}\right]=\frac{1}{\underline{\pi} \mathbf{D} \underline{e}} \cdot \underline{\pi}\left[(\mathbf{I}-\mathbf{C})^{-1} \mathbf{D}\right]^{h}(\mathbf{I}-\mathbf{C})^{-1} \underline{e} \\
& \mathrm{E}\left[A_{n}^{2}\right]=\frac{1}{\underline{\pi} \mathbf{D} \underline{e}}\left[2 \underline{\pi}(\mathbf{I}-\mathbf{C})^{-1} \underline{e}-1\right]
\end{aligned}
$$

Fig. 5 shows both correlation functions for Scene2. The period of the interarrival times is only 7 lags. Now, choose $\mathbf{C}$ and $\mathbf{D}$ such that the $\mathrm{MC}$ embedded at arrival instants has periodic states:

$$
\mathbf{C}:=\left(\begin{array}{cccc}
\mathbf{C}_{1} & \mathbf{0} & \cdots & \mathbf{0} \\
\mathbf{0} & \mathbf{C}_{2} & \ddots & \vdots \\
\vdots & & \ddots & \mathbf{0} \\
\mathbf{0} & \cdots & \mathbf{0} & \mathbf{C}_{T}
\end{array}\right), \quad \mathbf{D}:=\left(\begin{array}{cccc}
\mathbf{0} & \mathbf{D}_{1} & \cdots & \mathbf{0} \\
\vdots & \mathbf{0} & \ddots & \\
\mathbf{0} & & \ddots & \mathbf{D}_{T-1} \\
\mathbf{D}_{T} & \mathbf{0} & \cdots & \mathbf{0}
\end{array}\right)
$$

i.e. a period of $T$. The count of arrivals often does not show recorrelations, whereas the interarrival times do: Fig. 6. So a full characterization in terms correlation function needs both correlation functions.

\section{Performance of periodic models and exponentially decaying models}

By means of a queueing system representing an ATM multiplexer, models with periodic correlation function ("non-frame-buffered") and those with merely exponential decay 


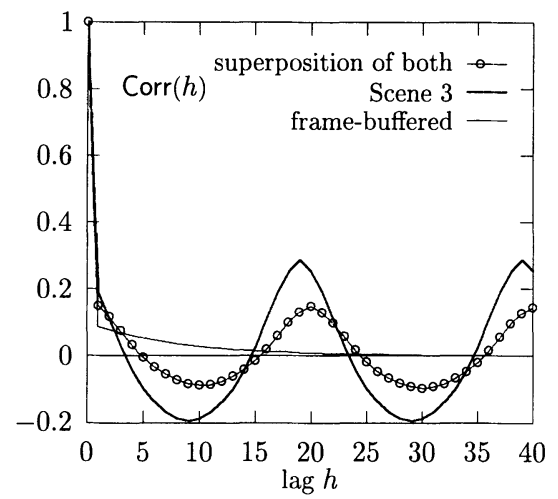

Fig. 3 Correlation functions: Scene 3, frame-buffered model and the superposition of both.

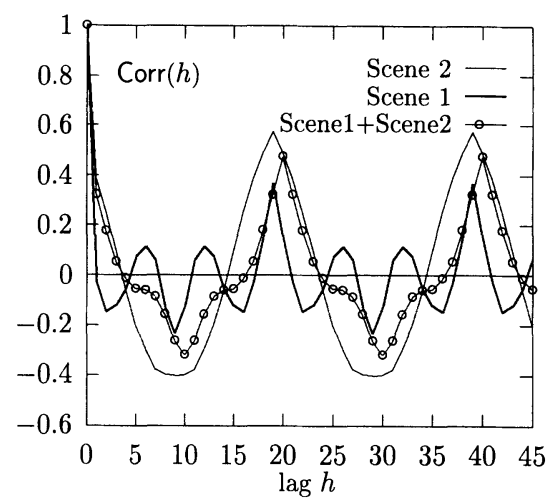

Fig. 4 Correlation functions: Scene 1, Scene 2 and the superposition of both.

("frame-buffered") are compared with respect to QOS parameters. We always consider two different streams and compute for each stream separately loss probabilities, mean and mean deviation of the waiting time.

This is done with the help of the queueing system $\mathrm{DMAP}_{1}^{\left[X_{1}\right]}+\mathrm{DMAP}_{2} / \mathrm{D} / 1 / s$, which is is solved according to the method described in [1] for the DBMAP/D/1/s-system: The deterministic service time is equal to the time unit ("slot"), there is a buffer of $s$ places and one server. The system works according to the "Departure First" rule: Arrivals and departures happen at the end of a slot and the departing cell leaves immediately before the arriving cell enters. The resulting system matrix is of M/G/1-type, see Appendix A Fig. 10). This matrix yields stationary state probabilities $\left[\underline{x}_{k}\right]_{i}, k=0, \ldots, s+1, i=1, \ldots, m$ that immediately after the end of a slot (taking a possible arrival or departure into account) there are $k$ cells in the system with the input process in phase $i$. An arriving batch at the end of the following slot therefore sees $\max (k-1,0)$ cells in the system. Having solved for the stationary state probabilities $\underline{x}_{k}, k=0, \ldots, s+1$, the per-stream QOSparameters loss probability, mean and mean deviation of the waiting time are obtained as follows (for details see Appendix A).

Whenever a batch of $v$ cells (" $v$-batch") arrives, QOS-parameters are defined as those of a test-cell marked among the cells of that batch, as it was done in [13]. The admission of a cell of the same batch is assumed to be totally random, so that the test-cell of a $v$-batch is chosen $\zeta$-th with probability $\frac{1}{v}$. Note that any cell, that is chosen first, is never lost (service time is equal to the time unit and Departure First rule!) and, therefore, a cell of stream 2 is not lost if only stream 2 generates an arrival. A detailed explanation of the formulae for per-stream loss probabilities and p.f.'s of the waiting time is given in the Appendix A.

\subsection{Loss probabilities}

Loss probability is defined here for

a) stream 1 by: $P$ \{stream 1 loses a test-cell given that the arriving batch contains a 


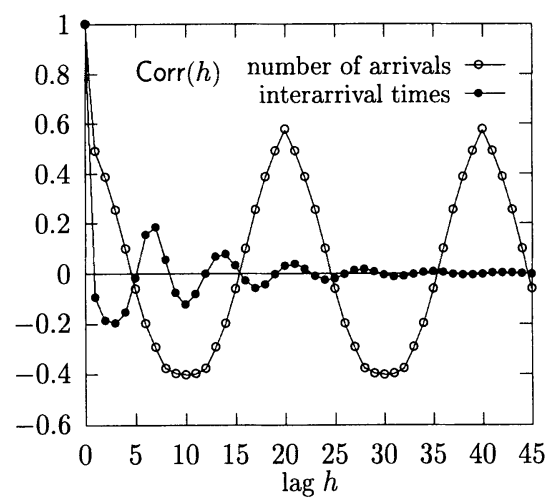

Fig. 5 Correlation functions of the count of arrivals and the interarrival times for Scene 2.

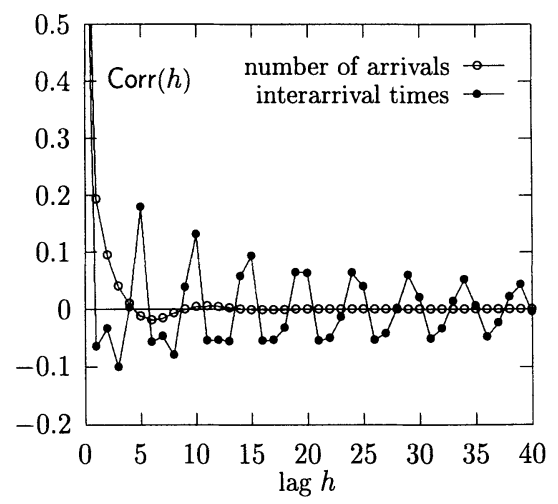

Fig. 6 No recorrelations of the count of arrivals, but recorrelations for the interarrival times. Period $T=5$.

test-cell of stream 1$\}$ (note that per definition, an arriving batch of stream 1 contains one and only one test-cell)

b) stream 2 by: $P$ \{stream 2 loses its cell given that the arriving batch contains a cell of stream 2 \}.

These definitions are consistent with the cell loss ratio: count of lost (test-)cells of a given stream devided by the count of (test-)cells generated by that stream. As shown in Appendix A, loss probability $P_{l o s s, \nu}$ for stream $\nu=1,2$ is computed by means of the expressions $\mathcal{T}_{1}$ and $\mathcal{T}_{12}$.

$$
\begin{aligned}
& P_{\text {loss }, 1}=\frac{\mathcal{T}_{1}+\mathcal{T}_{12}}{\underline{\pi}\left[\mathbf{D}^{(1)} \otimes\left(\mathbf{C}^{(2)}+\mathbf{D}^{(2)}\right)\right] \underline{e}} \\
& P_{\text {loss }, 2}=\frac{\mathcal{T}_{12}}{\underline{\pi}\left[\left(\mathbf{C}^{(1)}+\mathbf{D}^{(1)}\right) \otimes \mathbf{D}^{(2)}\right] \underline{e}} \\
& \quad \text { with } \underline{\pi}\left[\left(\mathbf{C}^{(1)}+\mathbf{D}^{(1)}\right) \otimes\left(\mathbf{C}^{(2)}+\mathbf{D}^{(2)}\right)\right]=\underline{\pi}, \text { and } \\
& \mathcal{T}_{1}:=\sum_{\mu=2}^{s+1} \sum_{v=s+3-\mu}^{s+1} \frac{v-(s+1-(\mu-1))}{v} \underline{x}_{\mu} \cdot\left(\mathbf{D}_{v}^{(1)} \otimes \mathbf{C}^{(2)}\right) \cdot \underline{e}+ \\
& +\sum_{v=s+2}^{b_{\text {max }}^{(1)}}\left[\frac{v-(s+1)}{v} \underline{x}_{0}\left(\mathbf{D}_{v}^{(1)} \otimes \mathbf{C}^{(2)}\right) \underline{e}+\sum_{\mu=1}^{s+1} \frac{v-(s+1-(\mu-1))}{v} \underline{x}_{\mu}\left(\mathbf{D}_{v}^{(1)} \otimes \mathbf{C}^{(2)}\right) \underline{e}\right] \\
& \mathcal{T}_{12}:=\sum_{\mu=2}^{s+1} \sum_{v=s+2-\mu}^{s} \frac{v+1-(s+1-(\mu-1))}{v+1} \underline{x}_{\mu} \cdot\left(\mathbf{D}_{v}^{(1)} \otimes \mathbf{D}^{(2)}\right) \cdot \underline{e}+
\end{aligned}
$$




$$
\begin{aligned}
& +\sum_{v=s+1}^{b_{\max }^{(1)}}\left[\frac{v+1-(s+1)}{v+1} \underline{x}_{0} \cdot\left(\mathbf{D}_{v}^{(1)} \otimes \mathbf{D}^{(2)}\right) \cdot \underline{e}+\right. \\
& \left.\quad+\sum_{\mu=1}^{s+1} \frac{v+1-(s+1-(\mu-1))}{v+1} \underline{x}_{\mu} \cdot\left(\mathbf{D}_{v}^{(1)} \otimes \mathbf{D}^{(2)}\right) \cdot \underline{e}\right]
\end{aligned}
$$

where $\mathbf{D}_{v}^{(1)}=x_{1}(v) \mathbf{D}^{(1)}$. The denominators represent the following probabilities:

$\underline{\pi}\left[\mathbf{D}^{(1)} \otimes\left(\mathbf{C}^{(2)}+\mathbf{D}^{(2)}\right)\right] \underline{e}=P\{$ stream 1 produces a test-cell $\}$ and

$\underline{\pi}\left[\left(\mathbf{C}^{(1)}+\mathbf{D}^{(1)}\right) \otimes \mathbf{D}^{(2)}\right] \underline{e}=P\{$ stream 2 produces a cell $\}$.

\subsection{Probability functions of the waiting time}

A single arriving cell sees a waiting time (in time slots), which is equal to the count of cells in the system. The test-cell of an arriving batch, that is chosen $\zeta$-th and is not lost, sees a waiting time equal to the count of cells in the system at the arrival instant plus the $\zeta-1$ cells (of the same batch), which were admitted before that cell. The cell at the end of the queue of the full system sees a waiting time of $s$ time slots so that the maximum waiting time is $s$. As shown in the appendix, the p.f.'s are composed of the functions $\mathcal{W}^{(\nu)}(\ell), \nu=1,2$ and $\mathcal{W}^{(12)}(\ell)$ :

$$
\begin{array}{ll}
P\left\{W_{1}=\ell\right\}=\frac{\mathbf{1}_{\ell=0}^{s}}{N_{1}}\left[\mathcal{W}^{(1)}(\ell)+\mathcal{W}^{(12)}(\ell)\right] & N_{1}=\sum_{\ell=0}^{s} P\left\{W_{1}=\ell\right\} \\
P\left\{W_{2}=\ell\right\}=\frac{\mathbf{1}_{\ell=0}^{s}}{N_{2}}\left[\mathcal{W}^{(12)}(\ell)+\mathcal{W}^{(2)}(\ell)\right] & N_{2}=\sum_{\ell=0}^{s} P\left\{W_{2}=\ell\right\}
\end{array}
$$

with

$$
\begin{aligned}
& \mathcal{W}^{(1)}(\ell):=\sum_{v=1}^{b_{\max }^{(1)}} \frac{1}{v} \sum_{\zeta=1}^{v}\left[\mathbf{1}_{\ell=\zeta-1}\left(\underline{x}_{0}+\underline{x}_{1}\right)+\mathbf{1}_{\ell=\zeta}^{s} \underline{x}_{\ell-\zeta+2}\right] \cdot\left(\mathbf{D}_{v}^{(1)} \otimes \mathbf{C}^{(2)}\right) \cdot \underline{e} \\
& \mathcal{W}^{(2)}(\ell):=\left[\mathbf{1}_{\ell=0}\left(\underline{x}_{0}+\underline{x}_{1}\right)+\mathbf{1}_{\ell=1}^{s} \underline{x}_{\ell+1}\right] \cdot\left(\mathbf{D}_{0}^{(1)} \otimes \mathbf{D}^{(2)}\right) \cdot \underline{e} \\
& \mathcal{W}^{(12)}(\ell):=\sum_{v=1}^{b_{\text {max }}^{(1)}} \frac{1}{v+1} \sum_{\zeta=1}^{v+1}\left[\mathbf{1}_{\ell=\zeta-1}\left(\underline{x}_{0}+\underline{x}_{1}\right)+\mathbf{1}_{\ell=\zeta}^{s} \underline{x}_{\ell-\zeta+2}\right] \cdot\left(\mathbf{D}_{v}^{(1)} \otimes \mathbf{D}^{(2)}\right) \cdot \underline{e}
\end{aligned}
$$

$\mathbf{1}$ denotes the indicator function: $\mathbf{1}_{\ell=a}^{b}:=1$ for $a \leq \ell \leq b$ and $=0$ else, $\mathbf{1}_{\ell=a}:=\mathbf{1}_{\ell=a}^{a}$; $\underline{x}_{k}:=\underline{0}$ for $k<0$.

\subsection{Numerical results for periodic or exponentially decaying correlation func- tions}

In the following examples, the scenes according to Table 1 were used. For the case of the frame-buffered model we used a "video source with uniform activity level" according to [1]. with the following parameters (in the notation used there): $M=10$ (count of phases), $\alpha=0.07926, \beta=0.0424, d=10.1$. Fig. 1,2 and 3 show the correlation function for this model with the label "frame-buffered" (and that of the superpsition with a 
a) Per-stream loss probabilities

\begin{tabular}{|c|c|c|c|c|c|c|c|c|}
\hline & \multicolumn{4}{|c|}{$b_{\max }^{(1)}=10$} & \multicolumn{4}{|c|}{$b_{\max }^{(1)}=2$} \\
\hline$s$ & Scene 1 & $\mathrm{GEO}^{[X]}$ & $\begin{array}{l}\text { frame- } \\
\text { buffered }\end{array}$ & $\mathrm{GEO}^{[X]}$ & Scene 1 & $\mathrm{GEO}^{[X]}$ & $\begin{array}{l}\text { frame- } \\
\text { buffered }\end{array}$ & $\mathrm{GEO}^{[X]}$ \\
\hline 10 & $4.445 \mathrm{e}-4$ & $\overline{8.316 \mathrm{e}-4}$ & $9.291 \mathrm{e}-4$ & $1.483 \mathrm{e}-3$ & $8.661 \mathrm{e}-6$ & $1.234 \mathrm{e}-5$ & $1.412 \mathrm{e}-4$ & $1.749 \mathrm{e}-4$ \\
\hline 15 & $3.828 \mathrm{e}-5$ & $7.216 \mathrm{e}-5$ & $1.258 \mathrm{e}-4$ & $1.982 \mathrm{e}-4$ & $6.916 \mathrm{e}-8$ & $9.779 \mathrm{e}-8$ & $6.732 \mathrm{e}-6$ & $8.322 \mathrm{e}-6$ \\
\hline 20 & $3.339 \mathrm{e}-6$ & $6.296 \mathrm{e}-6$ & $1.723 \mathrm{e}-5$ & $2.701 \mathrm{e}-5$ & $5.506 \mathrm{e}-10$ & \begin{tabular}{|c|}
$7.801 \mathrm{e}-10$ \\
\end{tabular} & $3.240 \mathrm{e}-7$ & $4.004 \mathrm{e}-7$ \\
\hline 30 & $2.558 \mathrm{e}-8$ & $4.816 \mathrm{e}-8$ & $3.254 \mathrm{e}-7$ & $5.088 \mathrm{e}-7$ & $3.477 \mathrm{e}-14$ & $4.924 \mathrm{e}-14$ & $9.308 \mathrm{e}-10$ & $7.532 \mathrm{e}-10$ \\
\hline
\end{tabular}

b) Per-stream mean waiting times in time slots (same tendency for mean deviation)

\begin{tabular}{|c|c|c|c|c|c|c|c|c|}
\hline & \multicolumn{4}{|c|}{$b_{\max }^{(1)}=10$} & \multicolumn{4}{|c|}{$b_{\max }^{(1)}=2$} \\
\hline$s$ & Scene 1 & $\mathrm{GEO}^{[X]}$ & $\begin{array}{l}\text { frame- } \\
\text { buffered }\end{array}$ & $\mathrm{GEO}^{[X]}$ & Scene 1 & $\mathrm{GEO}^{[X]}$ & $\begin{array}{l}\text { frame- } \\
\text { buffered }\end{array}$ & $\operatorname{GEO}^{[X]}$ \\
\hline 10 & 0.751 & 1.103 & $\overline{0.962}$ & 1.207 & 0.417 & 0.573 & 0.612 & 0.683 \\
\hline 15 & 0.783 & 1.139 & 1.026 & 1.269 & 0.417 & 0.573 & 0.619 & 0.689 \\
\hline 20 & 0.788 & 1.143 & 1.038 & 1.281 & 0.417 & 0.573 & 0.619 & 0.689 \\
\hline 30 & 0.788 & 1.144 & 1.040 & 1.283 & 0.418 & 0.573 & 0.619 & 0.689 \\
\hline
\end{tabular}

Table 2 QOS parameters for both streams of $G E O^{[X]}+D M A P / D / 1 / s$. DMAP represents Scene 1 (non-frame-buffered) or the frame-buffered model of [1].

non-frame-buffered periodic model).

The resulting arrival rate is 0.345 for a single stream in all cases. For a direct comparison of the frame-buffered and non-frame-buffered case, no batch random variable is used for the stream with recorrelations. Note that GEO is a DMAP with only one phase.

1. The GEO ${ }^{[X]}+\mathrm{DMAP} / \mathrm{D} / \mathbf{1} / s$ queue (Table 2). This case represents the situation that the existing traffic on a link is totally random and that a single DMAP representing one of the scenes considered above is superimposed. For all scenes the arrival rate was kept to be 0.345 . The maximum number $b_{m a x}^{(1)}$ of arrivals of the $\mathrm{GEO}^{[X]}$-stream was 2 or 10. Table 2 illustrates that as long as $b_{\max }^{(1)}$ is small in comparison with the buffer size $s$, there is a considerable difference in the QOS-parameters between periodic and exponentially decaying correlation function. The periodic case yields lower values. Similar results were found for scene 2 and 3 with the ordering of QOS-parameters: scene $1<$ scene $2,3<$ frame-buffered.

Used parameters: Period of the correlation function: 20, arrival rate of both streams: 0.345, mean arrival rate of the superposition: 0.730 , p.f. of the interarrival times of GEO: $a(k)=(1-q) q^{k-1} q=0.73$ for $b_{\max }^{(1)}=2$ and $q=0.8$ for $b_{\max }^{(1)}=10, \quad$ batchsize p.f. for $\mathrm{GEO}^{[X]}: x(v)=\left(1-q_{b}\right) q_{b}^{v-1} /\left(1-q_{b}^{b_{\text {max }}^{(1)}}\right), \quad q_{b}=0.4$.

2. The influence of the decay within the first period (Fig. 7). Using $\left(d_{1}, d_{1}, d_{2}, d_{2}, \ldots, d_{m}, d_{m}\right)$ instead of $\left(d_{1}, d_{2}, \ldots, d_{m}\right)$ for generating the DMAP with periodic correlation function both the form of the curve and the arrival rate remain unchanged, whereas the period is doubled, the decay from 1 becomes weaker and the first negative value of the correlation function appears for a higher lag $h$. Thus, a stretching by factor 2 is performed. Fig. 7 shows that loss probabilities for the $\mathrm{GEO}^{[X]}+\mathrm{DMAP} / \mathrm{D} / 1 / s$ queue 


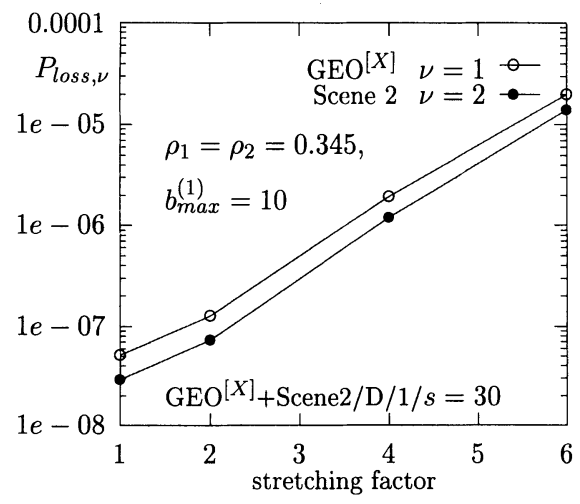

Fig. 7 Influence of the first lag with negative value of the correlation function on loss probablity (Scene 2 with different stretching factors).

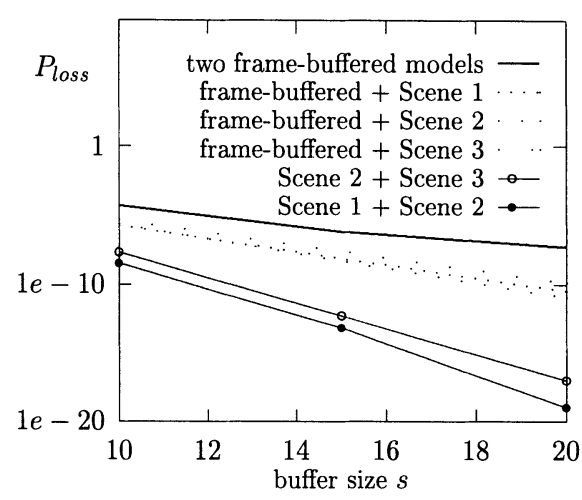

Fig. 8 Loss probabilities for different superpositions of streams (for both streams in a superposition $P_{\text {loss }}$ has approximately the same value).

with scene 2, when the stretching factor is enlarged in the way explained above, grow considerably. The same is true for the mean value and the mean deviation of the waiting time. From this, it can be concluded that the decay within the first period very much determines the QOS parameters. The higher distance between neighbouring recorrelation peaks, which is intuitively expected to reduce QOS parameters, obviously does not compensate for this effect. In the limit case, there are no negative values of the correlation function, as it is the case for frame-buffering. Loss probability of scene 2 for big stretching factors (e.g. 6) becomes higher than for the frame-buffered model (see Fig. 7 and Table 2), obviously because the absolute values of the correlation function of scene 2 with stretching factor 6 are then much higher than for the frame-buffered model, see Fig. 2.

3. The superposition of two individual video models (Fig. 8). Now the superposition of two of the scenes is considered. Again the arrival rate is kept to 0.345 for both streams. The results (Fig. 8) show a much bigger difference in the performance: Whenever there is among the superimposed sources a "frame-buffered" one, the loss probabilities grow clearly. There are little differences for the three scenes.

Remark: Superimposing two scenes without replacing the first row in $\mathbf{M}_{\text {cycle }}$ by $(0, a, b, 0, \ldots, 0)$ (this replacement results in an exponential decay of the recorrelation peaks, see section 3 ) yields a reducible matrix and cannot be solved by the algorithms of Kramer [10] or Grassmann et al. [6].

\subsection{Discussion of the results}

Frame-buffering is said to perform a "smoothing" of the original source data. This is true in the sense that bitrate fluctuations vanish within the length of one frame: instead of short periods of high bitrate followed by periods of low bitrate, for the duration of a frame the bitrate (and the cell rate) is constant (some mean value). However, this 
smoothing does not yield lower loss probability and waiting time, as it is shown by the above findings. And this is not surprising: The limit case of a CBR (Constant BitRate) source corresponds to "prebuffering the whole signal" and sending it with constant bitrate. It is widely agreed that multiplexing gain is much higher for VBR sources than for CBR ones. In terms of statistical quantities, the above findings can be explained by the fact that there are negative values of the correlation function for small lags $h$. This is in accordance with the examples in [8] where negative values in the coefficient of correlation of the interarrival times $(\operatorname{lag} h=1)$ yield lower loss probabilities than in the uncorrelated case.

\section{Conclusions}

By choosing the matrices $\mathbf{C}$ and $\mathbf{D}$ of a $\mathrm{DMAP}^{[X]}$ so that the $\mathrm{MC} \mathbf{C}+\mathbf{D}$ of the phase transitions has periodic states, periodic correlation functions of the count of arrivals in a test-interval (with recorrelations) can be obtained. It was possible to construct the correlation functions similar to those of the video scenes measured in $[7,15]$. However, a general method for determining the matrices $\mathbf{C}$ and $\mathbf{D}$ in order to obtain a given correlation function is not known and there seem to be limitations as to reconstruction of an arbitrary periodic curve with some symmetry properties. Using a batch size random variable reduces the peaks of the correlation function in most cases considerably. Correlation functions of superpositions of those scenes were computed.

The main conclusions are: QOS parameters are mainly depending on whether there are negative values of the correlation function for small lags $h$, and not so very much on the height of the recorrelation peaks. Periodic variations of the correlation function are worth exploiting in statistical multiplexing, if negative values of the correlation function occur (and they do as shown by the measurements in $[15,7]$ ).

\section{Appendix}

\section{A. Explaining formulae (15), (16), (17), and (18)}

Throughout this section consider a batch of $v \geq 1$ cells (" $v$-batch") due to stream 1 , a single cell due to stream 2 or both arriving at the end of a slot (slot of observation), Fig. 9. According to the "Departure First" rule, a departure (if any) happens immediately before the arrival. The occupancy seen by this arrival, is obtained from $\underline{x}_{\mu}, \mu=0,1, \ldots, s+1$

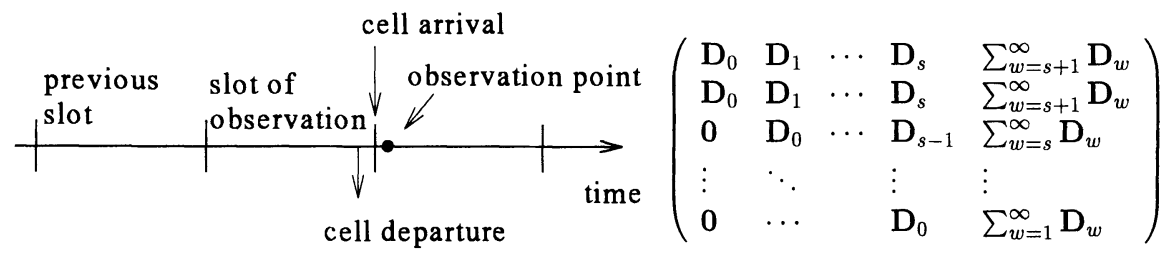

Fig. 9 Slot structure for "Departure First".

Fig. 10 System matrix for "Departure First" according to [1]. 
refering to the previous slot:

$\underline{x}_{\mu} \cdot\left(\mathbf{D}_{v}^{(1)} \otimes \mathbf{C}^{(2)}\right) \cdot \underline{e}=P\{$ stream 1 produces a $v$-batch, and stream 2 produces no arrival, and the arriving batch of $v$ cells sees $\max (\mu-1,0)$ cells in the system $\}$,

$\underline{x}_{\mu} \cdot\left(\mathbf{D}_{0}^{(1)} \otimes \mathbf{D}^{(2)}\right) \cdot \underline{e}=P\{$ stream 1 produces no arrival, and stream 2 produces a single arrival, and the arriving cell sees $\max (\mu-1,0)$ cells in the system $\}$,

$\underline{x}_{\mu} \cdot\left(\mathbf{D}_{v}^{(1)} \otimes \mathbf{D}^{(2)}\right) \cdot \underline{e}=P\{$ stream 1 produces a $v$-batch, and stream 2 produces an arrival, and the arriving batch of $v+1$ cells sees $\max (\mu-1,0)$ cells in the system $\}$.

Note that $\left[\underline{x}_{k}\right]_{i}, k=0, \ldots, s+1, i=1, \ldots, m$ means a stationary probability (i.e. it refers to an arbitrary slot) that immediately after the end of a slot (taking a possible arrival or departure into account and observing the "Departure First" rule ) there are $k$ cells in the system with the input process in phase $i$. Fig. 10 shows the system matrix.

The sequence of admitting the cells of an arriving batch to the system is assumed to be totally random. For characterizing the waiting time and loss probability of stream 1 we use, as done in [13], a test-cell marked among the cells of the arriving batch. The test-cell of a $v$-batch is chosen $\zeta$-th with probability $\frac{1}{v}$. If the arriving $(v+1)$-batch contains a cell of stream 2 and a $v$-batch of stream 1 , the cell of stream 2 is chosen $\zeta$-th with probability $\frac{1}{v+1}$, and the same is true for the test-cell of stream 1 .

A single cell (or the cell of a $v$-batch chosen first) arriving at the end of the slot of observation is never lost (due to the "Departure First" rule and the service time equal to the time unit, the system is never full at an arrival instant), and this cell sees a waiting time of $\max (\mu-1,0)$, if the occupancy was $\mu$ at end of the previous slot. (This is different for the "Arrival First" rule [17]!) A cell of an arriving batch chosen $\zeta$-th and not lost, sees a waiting time that is equal to the count of cells in the system plus the $\zeta-1$ cells of the same batch admitted before that cell.

\section{Loss probabilities}

1. Stream 1 loses a test-cell, no arrival due to stream 2: Imagine a $v$-batch of stream 1 arriving at the end of the slot of observation and stream 2 does not produce an arrival at that time instant. The sequence of admitting the cells of the batch to the queue is assumed to be totally random, i.e. the test-cell is admitted $\zeta$-th $(\zeta=1,2, \ldots, v)$, which we also denote by "the test-cell is admitted in position $\zeta$ ", with probability $\frac{1}{v}$. Let $L$ be the occupancy seen by the arriving batch. The test-cell can be lost only if $v>s+1-L$. In this case, the test-cell is lost, if it is chosen $(s+1-L+1)$-th, $(s+1-L+2)$-th, $\ldots, v$-th. So there are $v-(s+1-L)$ "positions" for the test-cell to be lost. For $\mathcal{T}_{1}:=P\{$ stream 1 produces an arrival, stream 2 produces no arrival, and test-cell of stream 1 is lost $\}$, we get:

$$
\mathcal{T}_{1}=\sum_{\mu=2}^{s+1} \sum_{v=s+3-\mu}^{s+1} \underbrace{\frac{v-(s+1-(\mu-1))}{v}}_{(\mathrm{ii})} \underbrace{x_{\mu} \cdot\left(\mathbf{D}_{v}^{(1)} \otimes \mathbf{C}^{(2)}\right) \cdot \underline{e}}_{(\mathrm{i})}+
$$

batch is not bigger than system capacity and test-cell is lost

$$
+\underbrace{\sum_{v=s+2}^{b_{\max }^{(1)}}\left[\frac{v-(s+1)}{v} \underline{x}_{0}\left(\mathbf{D}_{v}^{(1)} \otimes \mathbf{C}^{(2)}\right) \underline{e}+\sum_{\mu=1}^{s+1} \frac{v-(s+1-(\mu-1))}{v} \underline{x}_{\mu}\left(\mathbf{D}_{v}^{(1)} \otimes \mathbf{C}^{(2)}\right) \underline{e}\right]}
$$

batch is bigger than the capacity $s+1$ of the system and test-cell is lost 


\begin{abstract}
Hints:
(i): Occupancy at the slot of observation is $\max (\mu-1,0)$, only stream 1 produces a $v$-batch (ii): $v-(s+1-(\mu-1))$ loss positions for the test-cell, each position has probability $1 / v$. (iii): Occupancy at the slot of observation is $\max (\mu-1,0)$, stream 1 produces a $v$-batch, stream 2 a single cell

(iv): $v+1-(s+1-(\mu-1))$ loss positions for the test-cell (or cell of stream 2), each position has probability $1 /(v+1)$.
\end{abstract}

2. Both streams produce an arrival: If stream 1 produces a $v$-batch and stream 2 produces a single cell, loss of stream 1 is described by refering to its test-cell (now with respect to a $(v+1)$-batch). The admission of the cell of stream 2 is also totally random so that there is the same count of loss positions for this cell and the test-cell. Therefore $P$ \{stream 1 produces an arrival, and stream 2 produces an arrival, and test-cell of stream 1 is lost $\}$ is equal to $P$ \{stream 1 produces an arrival, and stream 2 produces an arrival, and cell of stream 2 is lost $\}$ and denoting this probability by $\mathcal{T}_{12}$, it is:

$$
\mathcal{T}_{12}:=\quad \sum_{\mu=2}^{s+1} \sum_{v=s+2-\mu}^{s} \underbrace{\frac{v+1-(s+1-(\mu-1))}{v+1}}_{\text {(iv) }} \underbrace{x_{\mu} \cdot\left(\mathbf{D}_{v}^{(1)} \otimes \mathbf{D}^{(2)}\right) \cdot \underline{e}}_{\text {(iii) }} \quad+
$$

$v$-batch of stream 1 plus cell of stream 2 is less or equal to system capacity $s+1$

$$
+\underbrace{\sum_{v=s+1}^{b_{\max }^{(1)}}\left[\frac{v+1-(s+1)}{v+1} \underline{x}_{0}\left(\mathbf{D}_{v}^{(1)} \otimes \mathbf{D}^{(2)}\right) \underline{e}+\sum_{\mu=1}^{s+1} \frac{v+1-(s+1-(\mu-1))}{v+1} \underline{x}_{\mu}\left(\mathbf{D}_{v}^{(1)} \otimes \mathbf{D}^{(2)}\right) \underline{e}\right]}
$$

$v$-batch of stream 1 plus cell of stream 2 is more than system capacity $s+1$

Since a cell of stream 2 is never lost, if stream 2 produces an arrival alone, the Eqs. (15) and (16) follow; the denominators are necessary since loss probability is a conditional probability.

\title{
Waiting times
}

3. Contribution to the waiting time of stream 1 , if stream 1 produces a batch and stream 2 no arrival: (The indicator function 1 is defined under formulae (17), (18).)

$$
\mathcal{W}^{(1)}(\ell):=\sum_{v=1}^{b_{\max }^{(1)}} \sum_{\zeta=1}^{v} \underbrace{\frac{1}{v}}_{(\mathrm{v})}[\underbrace{\mathbf{1}_{\ell=\zeta-1}\left(\underline{x}_{0}+\underline{x}_{1}\right)}_{(\mathrm{vi})}+\underbrace{\mathbf{1}_{\ell=\zeta}^{s} \underline{x}_{\ell-\zeta+2}}_{(\mathrm{vii})}] \cdot\left(\mathbf{D}_{v}^{(1)} \otimes \mathbf{C}^{(2)}\right) \cdot \underline{e}
$$

4. Contribution to the waiting time of stream 2 , if stream 2 produces an arrival and stream 1 no arrival:

$$
\mathcal{W}^{(2)}(\ell):=[\underbrace{\mathbf{1}_{\ell=0}\left(\underline{x}_{0}+\underline{x}_{1}\right)}_{(\text {viii })}+\underbrace{\mathbf{1}_{\ell=1}^{s} \underline{x}_{\ell+1}}_{(\mathrm{ix})}] \cdot\left(\mathbf{D}_{0}^{(1)} \otimes \mathbf{D}^{(2)}\right) \cdot \underline{e}
$$




\section{Hints:}

(v): Probability that test-cell is chosen $\zeta$-th.

(vi): Arriving $v$-batch sees an empty system, its test-cell chosen $\zeta$-th contributes to a waiting time of $\ell=\zeta-1$.

(vii): Arriving $v$-batch sees $\ell-(\zeta-1) \geq 0$ cells in the system (at the end of the previous slot the occupancy was $\ell-\zeta+2$ ), test-cell chosen $\zeta$-th contributes to a waiting time of $\ell$.

(viii): Cell of stream 2 sees an empty system and contributes to the waiting time of $\ell=0$.

(ix): Cell of stream 2 sees an occupancy of $\ell$ and thus contributes to a waiting time of $\ell$.

(x): Probability that test-cell of stream 1 (cell of stream 2) is chosen $\zeta$-th.

(xi): Arriving $(v+1)$-batch sees an empty system, test-cell of stream 1 (cell of stream 2) chosen $\zeta$-th contributes to a waiting time of $\ell=\zeta-1$.

(xii): Arriving $(v+1)$-batch sees $\ell-(\zeta-1) \geq 0$ cells in the system (at the end of the previous slot the occupancy was $\ell-\zeta+2$ ), test-cell of stream 1 (cell of stream 2) chosen $\zeta$-th contributes to a waiting time of $\ell$.

5. Contribution to the waiting time of stream 1 resp. 2, if both streams produce arrivals

$\mathcal{W}^{(12)}(\ell):=\sum_{v=1}^{b_{\max }^{(1)}} \sum_{\zeta=1}^{v+1} \underbrace{\frac{1}{v+1}}_{(\mathrm{x})}[\underbrace{\mathbf{1}_{\ell=\zeta-1}\left(\underline{x}_{0}+\underline{x}_{1}\right)}_{(\mathrm{xi})}+\underbrace{\mathbf{1}_{\ell=\zeta}^{s} \underline{\underline{x}}_{\ell-\zeta+2}}_{(\mathrm{xii})}] \cdot\left(\mathbf{D}_{v}^{(1)} \otimes \mathbf{D}^{(2)}\right) \cdot \underline{e}$

In order to get a correct probability function (p.f.), one needs the normalizing constants $N_{1}$ and $N_{2}$ in Eqs. (17) and (18).

B. Measured correlation functions cited from [7]
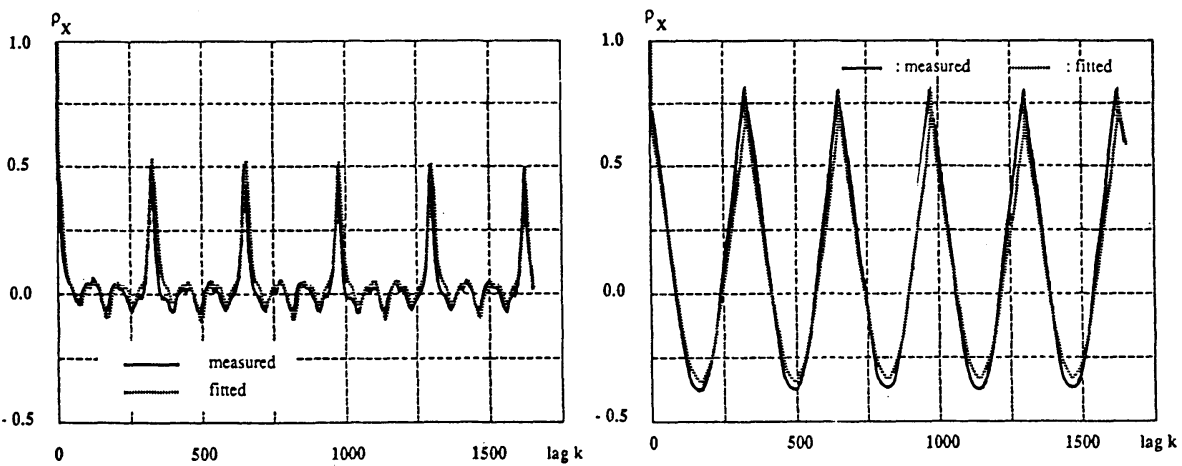

Fig. 11 Measured correlation functions for VBR video [7]. 


\section{REFERENCES}

1. C. Blondia and O. Casals. Statistical multiplexing of VBR sources: A matrix-analytic approach. Perf. Eval., Vol. 16, No. 1-3, pp. 5-20, Nov. 1992.

2. G.E.P. Box, G.M. Jenkins. Time Series Analysis (forecasting and control). Holden Day, Oakland, 1976.

3. E. Cinlar. Introduction to Stochastic Processes. Prentice Hall, New Jersey, 1st edition, 1975.

4. J. Cosmas, A. Odinma-Okafor. Characterization of Variable Rate Video Codecs in ATM to a geometrically modulated deterministic process model. In ITC-13, Vol. 14, Copenhagen, pp. 773-780, June 19-26 1991.

5. D.R. Cox, H.D. Miller. The Theory of Stochastic Processes. Chapman \& Hall, London, 3rd reprint, 1972.

6. W. K. Grassmann, M. Taksar, D. Heyman. Regenerative Analysis and Steady State Distributions for Markov Chains. Opns. Res., Vol. 33, No. 5, pp. 1107-1116, Sept.-Okt. 1985.

7. R. Grünenfelder J.P. Cosmas, S. Manthorpe, A. Odinma-Okafor. Characterization of Video Codecs as Autoregressive Moving Average Processes and Related Queueing System Performance. IEEE J. Sel. Areas Com, Vol. 9, No. 3, pp. 284-293, April 1991.

8. C. Herrmann. Analysis of the Discrete-time SMP/D/1/s Finite Buffer Queue with Applications in ATM. In IEEE INFOCOM'93, pp. 160-167, Session 2a.3, San Francisco, March 28 - April 1, 1993.

9. R. Kishimoto, Y. Ogata, F. Inumaru. Generation Interval Distribution Characteristics of Packetized Variable Rate Video Coding Data Streams in an ATM Network. IEEE J. Sel. Areas in Com, Vol. 7, No. 5, pp. 833-841, June 1989.

10. M. Kramer. Computational Methods for Markov Chains occuring in Queueing Theory. In Messung, Modellierung und Bewertung von Rechnersystemen, Informatik Fachberichte 154, Springer, pp.164-175, 1987.

11. D.M. Lucantoni. New Results on the single Server Queue with a Batch Markovian Arrival Process. Stochastic Models, Communications in Statistics, Vol. 7, No. 1, pp. 1-46, 1991.

12. B. Maglaris, D. Anastassiou, P. Sen, G. Karlsson, J.D. Robbins. Performance Models of Statistical Multiplexing in Packet Video Communications. IEEE Trans Com., Vol. 36, No. 7, pp. 834-843, July 1988.

13. M. Murata et al. Analysis of a Discrete-Time Single-Server Queue with Bursty Inputs for Traffic Control in ATM Networks. IEEE J-SAC, Vol. 8, No. 3, pp. 447-458, April 1990.

14. M. Nomura, T. Fujii, N. Ohta. Basic Characteristics of Variable Video Coding in ATM Environment. IEEE J-SAC, Vol. 7, No. 5, pp. 752-760, June 1989.

15. A. Odinma-Okafor. The Characterization of Variable Rate Video Signals. PhD thesis, Dep. of Electronic Eng., Queen Mary \& Westfield College, London, Oct. 1991.

16. P. Sen, B. Maglaris, N. Rikli, D. Anastassiou. Models for Packet Switching of Variable Rate Video Sources. IEEE J. on Sel. Areas in Com., Vol. 7, No. 5, pp. 865-869, 1989.

17. T. Takine, T. Suda, T Hasegawa. Cell Loss and Output Process Analysis of a Finite-Buffer Discrete-time ATM Queueing System with Correlated Arrivals. In IEEE INFOCOM'93, pp. 1259-1269, paper 10C.3.1, San Francisco, March 30-April 1, 1993. 